ARTICLE

https://doi.org/10.1038/s41467-018-08206-6

\title{
A welding phenomenon of dissimilar nanoparticles in dispersion
}

Zhiqi Huang (10 1,2, Zhi-Jian Zhao (1) 1, Qian Zhang ${ }^{2}$, Lili Han³ ${ }^{3}$ Xiumei Jiang ${ }^{4}$, Chao Li ${ }^{3}$, Maria T. Perez Cardenas², Peng Huang (10 ${ }^{5}$, Jun-Jie Yin (10) ${ }^{4}$, Jun Luo ${ }^{3}$, Jinlong Gong (1) ${ }^{1} \&$ Zhihong Nie (i) ${ }^{2,6}$

The oriented attachment of small nanoparticles (NPs) is recognized as an important mechanism involved in the growth of inorganic nanocrystals. However, non-oriented attachment of dissimilar NPs has been rarely observed in dispersion. This communication reports a welding phenomenon occurred directly between as-synthesized dispersions of single-component Au and chalcogenide NPs, which leads to the formation of asymmetric Au-chalcogenide hybrid NPs (HNPs). The welding of dissimilar NPs in dispersion is mainly driven by the ligand desorption-induced conformal contact between NPs and the diffusion of Au into chalcogenide NPs. The welding process can occur between NPs with distinct shapes or different capping agents or in different solvent media. A two-step assembly-welding mechanism is proposed for this process, based on our in situ electron spin resonance measurements and $a b$ initio molecular dynamics simulation. The understanding of NP welding in dispersion may lead to the development of unconventional synthetic tools for the fabrication of hybrid nanostructures with diverse applications.

\footnotetext{
${ }^{1}$ Key Laboratory for Green Chemical Technology of Ministry of Education, School of Chemical Engineering and Technology, Collaborative Innovation Center of Chemical Science and Engineering, Tianjin University, 300072 Tianjin, China. ${ }^{2}$ Department of Chemistry and Biochemistry, University of Maryland, College Park, MD 20742, USA. ${ }^{3}$ Center for Electron Microscopy TUT-FEl Joint Laboratory, Institute for New Energy Materials \& Low-Carbon Technologies; School of Materials Science and Engineering, Tianjin University of Technology, 300384 Tianjin, China. ${ }^{4}$ Division of Analytical Chemistry, Office of Regulatory Science, Center for Food Safety and Applied Nutrition, U.S. Food and Drug Administration, College Park, MD 20740, USA. ${ }^{5}$ Guangdong Key Laboratory for Biomedical Measurements and Ultrasound Imaging, Department of Biomedical Engineering, School of Medicine, Shenzhen University, 518060 Shenzhen, China. ${ }^{6}$ State Key Laboratory of Molecular Engineering of Polymers, Department of Macromolecular Science, Fudan University, Shanghai, 200438 P.R.,

China. Correspondence and requests for materials should be addressed to J.G. (email: jlgong@tju.edu.cn) or to Z.N. (email: znie@umd.edu)
} 
C olloidal inorganic hybrid nanoparticles (NPs) (HNPs) are multicomponent colloids with dissimilar inorganic components integrated within one colloidal particle. Compared with single-component NPs, HNPs not only combine chemical and physical properties of each component, but also may exhibit new synergistic properties that are not attainable by any of their individual constituent components ${ }^{1-4}$. As a result, HNPs have outperformed single-component NPs in a variety of applications ranging from energy ${ }^{5-7}$ to biomedicine ${ }^{8}$. Recently, enormous progress has been made in the wet-chemical synthesis of HNPs with different material combinations ${ }^{9-11}$. The dominating mechanism involved in such synthesis is the so-called seed-mediated growth in which a secondary component monomer nucleates on the surface of a primary component seed ${ }^{12,13}$. During the synthesis, a delicate control over the growth kinetics and interfacial energy between multiple components is typically required to obtain HNPs with desired topology and morphology ${ }^{14-16}$. This, however, represents a synthetic challenge for achieving reproducible and scalable production of HNPs. Therefore, there is an urgent need to develop novel synthetic approaches beyond seeded-mediated growth for the synthesis of high-quality HNPs.

The oriented attachment of small NPs (e.g., semiconductors, metals) is recognized as an important mechanism involved in the growth of inorganic nanocrystals ${ }^{17}$. Recently, a broad range of complex nanostructures such as one-dimensional nanowires (NWs) $^{18,19}$ and two-dimensional nanosheets ${ }^{20,21}$ has been synthesized via the oriented attachment. The welding of NPs to form larger nanostructures in this process is largely driven by the instability of NPs and the elimination of common crystal facets of neighboring nanocrystals. This phenomenon, however, has been usually limited to the welding of NPs of the same kind, due to the general requirement on lattice matching. In contrast, the nonoriented attachment mechanism not only occurs between the same type of NPs ${ }^{22,23}$, but also allows for the welding of dissimilar NPs, thus facilitating the synthesis of HNPs with diverse compositions. For example, the welding of $\mathrm{Au}$ and $\mathrm{Ag}$ NPs was observed upon contact in vacuum at room temperature ${ }^{24}$. However, the nonoriented attachment between dissimilar NPs in dispersion has rarely been reported. One example involves the random aggregation and uncontrollable welding of CuSe and $\operatorname{In}_{2} \mathrm{~S}_{3}$ NPs in dispersion to produce single-component $\mathrm{CuInS}_{x} \mathrm{Se}_{1-x} \mathrm{NPs}$ with irregular shapes ${ }^{25}$, rather than multicomponent hybrid nanostructures with distinct domains. To date, there has been no report on the successful welding of dissimilar NPs in dispersions to synthesize multicomponent HNPs.

Here, we describe a welding phenomenon occurred directly between as-synthesized single-component $\mathrm{Au}$ and chalcogenide (e.g., $\mathrm{Ag}_{2} \mathrm{~S}, \mathrm{Ag}_{2} \mathrm{Se}$ ) NPs in dispersion, which leads to the formation of asymmetric $\mathrm{Au}$-chalcogenide HNPs. This welding process does not require lattice matching between NPs and exhibited obvious atom diffusion, which are characteristics of non-oriented attachment mechanism. The diffusion of $\mathrm{Au}$ into $\mathrm{Ag}_{2} \mathrm{~S}$ domains is observed in the derived HNPs and is believed to be the driven force of the welding process ${ }^{26}$. This mechanism is further confirmed by molecular dynamic simulation, which shows the penetration of $\mathrm{Au}$ atoms into $\mathrm{Ag}_{2} \mathrm{~S}$ lattice at the $\mathrm{Au}-\mathrm{Ag}_{2} \mathrm{~S}$ interface. The welding process is found to be largely dependent on the ligand desorption that brings dissimilar NPs into close proximity. We monitor the welding process in situ by quantifying the photoreduction of a nitroxyl radical 2,2,6,6-tetramethylpiperidine-1-oxyl (TEMPO) using electron spin resonance (ESR) spectroscopy. This wedding phenomenon is also observed between NPs with distinct shapes. When Au nanorods (NRs) and $\mathrm{Au}$ NWs are used, their welding with spherical $\mathrm{Ag}_{2} \mathrm{~S}$ NPs produce matchstick-like or tree burl-like HNPs. Through the current study, we have demonstrated the merging of dissimilar NPs in dispersions to form multicomponent HNPs with defined shapes and morphologies by controlling the ligands on the surface of initial NPs. We have also identified a new two-step assembly-diffusion mechanism for the production of HNPs, which distinguishes our study from others. This work represents an important step toward bottom-up synthesis of complex multicomponent HNPs with desired morphologies and properties via controlled NP welding.

\section{Results}

Welding of spherical $\mathbf{A u}$ and $\operatorname{Ag}_{2} \mathrm{~S}$ NPs. Spherical Au NPs of $14.0 \pm 1.3 \mathrm{~nm}$ in diameter were prepared by a citrate-reduction method $^{27}$ (Supplementary Fig. 1) and surface-modified with thiol-terminated poly (ethylene glycol) (PEG-SH, $M_{\mathrm{w}}=5 \mathrm{k}$ ) with controlled density (Au-1 to Au-4, Supplementary Table 1). Spherical $\mathrm{Ag}_{2} \mathrm{~S}$ NPs of $16.0 \pm 2.9 \mathrm{~nm}$ in diameter were synthesized using a bovine serum albumin (BSA)-directed growth method 28 (Supplementary Fig. 2) and were washed several times to remove excess BSA before use. Au NPs with low density of PEG and $\mathrm{Ag}_{2} \mathrm{~S}$ NPs were then mixed in a predetermined ratio and incubated at ambient condition for $24 \mathrm{~h}$ to allow for the welding. Figure la shows the representative transmission electron microscopy (TEM) image of the derived oligomer-like $\mathrm{Au}-\mathrm{Ag}_{2} \mathrm{~S}$ HNPs. All the $\mathrm{Ag}_{2} \mathrm{~S}$ NPs are attached to Au NPs to form HNPs in which $\mathrm{Au}$ and $\mathrm{Ag}_{2} \mathrm{~S}$ domains can be readily distinguished (dark: Au; gray: $\mathrm{Ag}_{2} \mathrm{~S}$ ). The 0.23 and $0.31 \mathrm{~nm} \mathrm{~d}$-spacing can be assigned to the (111) facet of $\mathrm{Au}$ and (121) facet of $\mathrm{Ag}_{2} \mathrm{~S}$, respectively (Fig. 1b). High-resolution TEM (HRTEM) image (Fig. 1b) and high-angle annular dark-field scanning TEM (HAADF-STEM) image (Fig. 1c) of an individual welded $\mathrm{Au}-\mathrm{Ag}_{2} \mathrm{~S}$ HNP clearly show that the $\mathrm{Au}$ and $\mathrm{Ag}_{2} \mathrm{~S}$ domains are fused together rather than physically attached or overlapped due to drying effect. In between the $\mathrm{Au}$ and $\mathrm{Ag}_{2} \mathrm{~S}$ domain, there is a diffusion region where $\mathrm{Au}$ gradually diffuses into $\mathrm{Ag}_{2} \mathrm{~S}$ domain, as indicated by the spread of dark $\mathrm{Au}$ domain into gray $\mathrm{Ag}_{2} \mathrm{~S}$ domain. The successful welding of $\mathrm{Au}$ and $\mathrm{Ag}_{2} \mathrm{~S}$ NPs in dispersion was also confirmed by the redshift of the plasmonic peak of Au NPs from 510 to $525 \mathrm{~nm}$ in the UV/Vis spectroscopy analysis (Supplementary Fig. 3). The spectra of $\mathrm{Au}$ and $\mathrm{Ag}_{2} \mathrm{~S}$ mixture $(0,2$, and $24 \mathrm{~h})$ showed strong absorption below $500 \mathrm{~nm}$, compared with that of pure Au NP dispersion. This is due to the summation of the absorption of $\mathrm{Au}$ and $\mathrm{Ag}_{2} \mathrm{~S}$ NPs. Moreover, upon mixing, the electrostatic interaction between oppositely charged $\mathrm{Au}$ and $\mathrm{Ag}_{2} \mathrm{~S}$ NPs immediately induced the assembly of dissimilar NPs and brought the $\mathrm{Ag}_{2} \mathrm{~S}$ NPs to the close proximity of Au NPs. The red-shift of the absorption spectra can thus be ascribed to the increase of local refractive index around $\mathrm{Au} \mathrm{NPs}$ after $\mathrm{Ag}_{2} \mathrm{~S} \mathrm{NP}$ attachment ${ }^{29}$.

We note that the number of $\mathrm{Au}$ and $\mathrm{Ag}_{2} \mathrm{~S}$ domains within the welded HNPs does not exactly follow the feeding ratio of building blocks. As an example, at 1:1 ratio (volume of NP dispersion), the yield of dimers composed of one $\mathrm{Au}$ and one $\mathrm{Ag}_{2} \mathrm{~S}$ domain (Fig. 1d) is $\sim 55 \%$. HNPs with other morphologies were also present in the product: $27 \%$ of trimers composed of two Au and one $\mathrm{Ag}_{2} \mathrm{~S}$ domains (Fig. 1e), 9\% of tetramers composed of three $\mathrm{Au}$ and one $\mathrm{Ag}_{2} \mathrm{~S}$ domains, 6\% of trimers composed of one $\mathrm{Au}$ and two $\mathrm{Ag}_{2} \mathrm{~S}$ domains (Fig. 1f), and 3\% of tetramers composed of one $\mathrm{Au}$ and three $\mathrm{Ag}_{2} \mathrm{~S}$ domains (Fig. 1g). We attribute this relatively wide distribution of HNPs to the probability of collision and contact between disparate NPs in dispersion. After the formation of dimers with one $\mathrm{Au}$ and one $\mathrm{Ag}_{2} \mathrm{~S}$ domains, they may further interact with either $\mathrm{Au}$ or $\mathrm{Ag}_{2} \mathrm{~S} \mathrm{NPs}$ in the system to form HNPs with more than two domains. Nevertheless, the product distribution could be roughly tuned by varying the feeding ratio of dissimilar NPs. As shown in Fig. 1h, increasing 

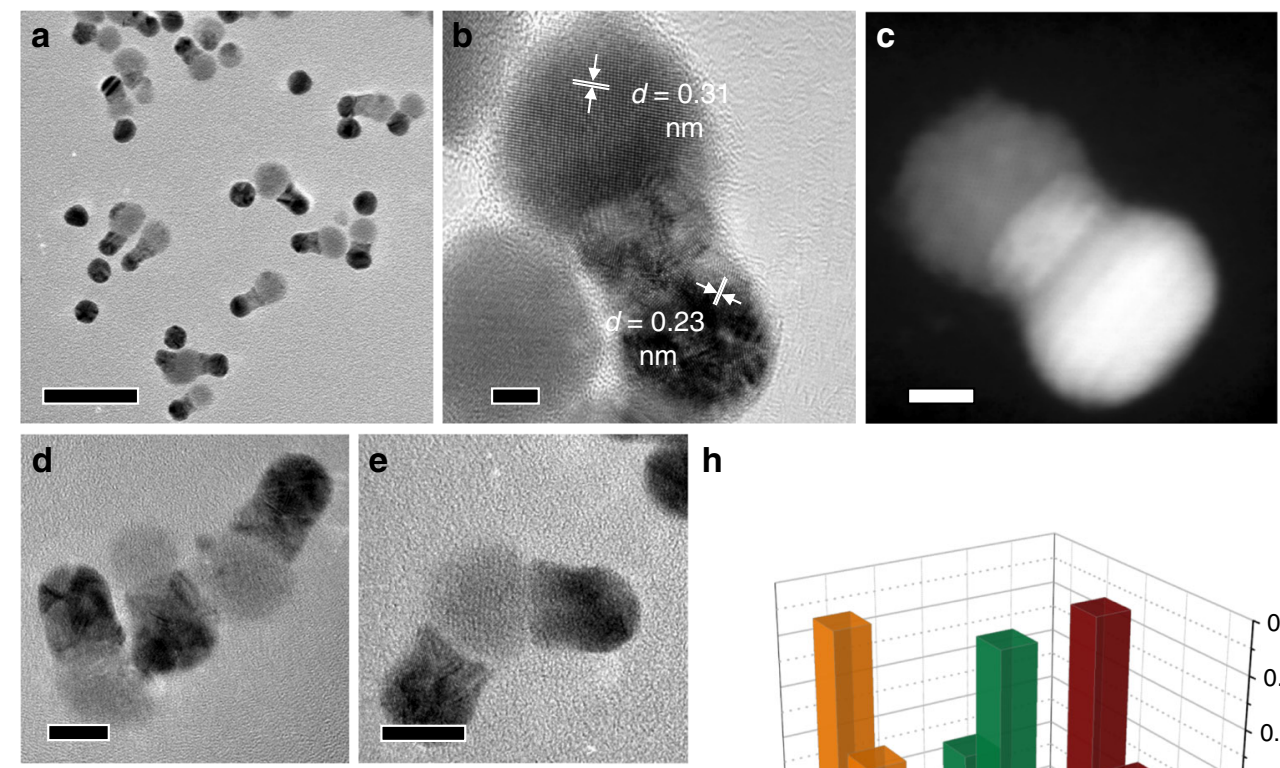

h
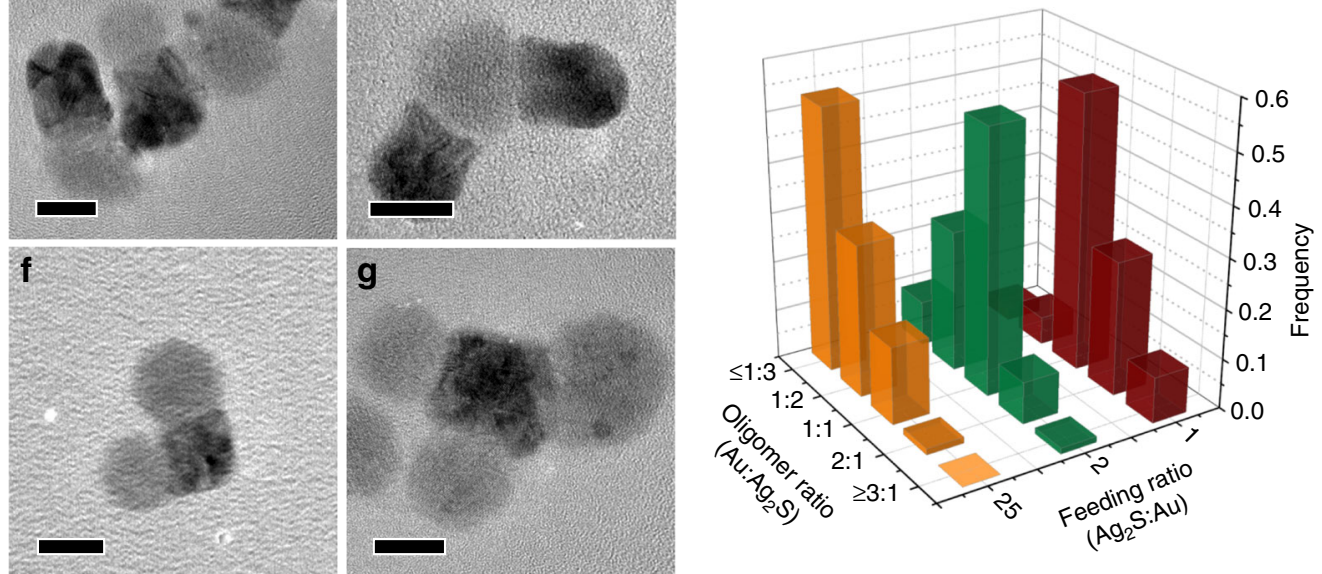

Fig. 1 Formation of $\mathrm{Au}-\mathrm{Ag}_{2} \mathrm{~S}$ oligomer-like HNPs through welding of spherical $\mathrm{Au}$ and $\mathrm{Ag}_{2} \mathrm{~S} N \mathrm{NPs}$. a TEM image of welded $\mathrm{Au}-\mathrm{Ag}_{2} \mathrm{~S} H N P s, \mathbf{b} H R T E M$, and $\mathbf{c}$ HAADF-STEM images of individual $A u-A_{2} S$ dimer formed by welding $A u$ and $\mathrm{Ag}_{2} \mathrm{~S} N \mathrm{NPs}_{\mathrm{s}}$ in dispersion. $\mathbf{d}-\mathbf{g}$ TEM images of $A u-A g_{2} \mathrm{~S} H N \mathrm{Hs}_{\mathrm{s}}$ with different $\mathrm{Au}: \mathrm{Ag}_{2} \mathrm{~S}$ domain ratios: $\mathbf{d} 1: 1, \mathbf{e} 2: 1, \mathbf{f} 1: 2$, and $\mathbf{g} 1: 3$. h Statistical histogram showing the $A \mathrm{u}: \mathrm{Ag}_{2} \mathrm{~S}$ domain ratio of derived $\mathrm{Au}-\mathrm{Ag}_{2} \mathrm{~S} H N P s$ at different feeding ratios of $\mathrm{Ag}_{2} \mathrm{~S}$ :Au NPs. Scale bars are $50 \mathrm{~nm}$ in $\mathbf{a}, 5 \mathrm{~nm}$ in $\mathbf{b}$, $\mathbf{c}$, and $10 \mathrm{~nm}$ in $\mathbf{d}-\mathbf{g}$

the feeding ratio of $\mathrm{Ag}_{2} \mathrm{~S}: \mathrm{Au}$ NPs led to the formation of HNPs with a larger number of $\mathrm{Ag}_{2} \mathrm{~S}$ domains in average within individual HNPs (Supplementary Fig. 4).

The grafting of PEG on Au NP surface is essential for stabilizing the colloidal particles during their welding in dispersion. More importantly, the grafting density of PEG determines the onset and rate of welding process. Without PEG modification $(\mathrm{Au}-0)$, the negatively charged Au NPs (zeta potential of $-43 \mathrm{mV}$ ) strongly attract positively charged $\mathrm{Ag}_{2} \mathrm{~S}$ NPs (zeta potential of $+32 \mathrm{mV}$ ), thus leading to irreversible aggregation and precipitation of these NPs in dispersion ${ }^{30}$ (Supplementary Fig. 5a). The presence of PEG weakened the surface charges on the NPs (Au-1,2): the absolute values of zeta potential of NPs decreased significantly from about -45 to $-15 \mathrm{mV}$ with increasing PEG coverage on Au NPs from 0 to $\sim 0.7$ chains $\mathrm{nm}^{-2}$ (Supplementary Fig. 6). This can be explained by the replacement of negatively charged citrate by neutral PEG and the burying of charged citrate under the PEG layer. It is worth noting that the welding only occurs for Au NPs with a moderate polymer coverage $\left(\sim 0.05-0.11\right.$ chain $\left.\mathrm{nm}^{-2}\right)$ (Supplementary Table 1 and Supplementary Fig. 5b). A high density of PEG (> 0.36 chain $\mathrm{nm}^{-2}$ ) hinders the welding process (Supplementary Table 1 and Supplementary Fig. 5c), as covalently bonded PEG chains are stable on Au surface and barely drop off from $\mathrm{Au}$ surfaces ${ }^{31,32}$.

Mechanism investigation of the welding process. In contrast to pristine $\mathrm{Au}$ and $\mathrm{Ag}_{2} \mathrm{~S}$ NPs, the presence of a Schottky barrier at the $\mathrm{Au}-\mathrm{Ag}_{2} \mathrm{~S}$ interface promotes the hot electron-hole separation and prolongs the lifetime of charge carriers in photo-irradiated
$\mathrm{Au}-\mathrm{Ag}_{2} \mathrm{~S}$ HNPs. The photo-generated hot electrons can be detected by ESR spectroscopy using nitroxyl radical TEMPO as a probe $^{33,34}$. We compared the loss of TEMPO (i.e., reduction of TEMPO by hot electrons) in the dispersion of $\mathrm{Au} \mathrm{NPs,} \mathrm{Ag}_{2} \mathrm{~S} \mathrm{NPs}$, and $\mathrm{Au}-\mathrm{Ag}_{2} \mathrm{~S}$ HNPs upon irradiation with visible light. As shown in Fig. 2a, the loss of TEMPO was significant in welded $\mathrm{Au}-\mathrm{Ag}_{2} \mathrm{~S}$ HNPs. In contrast, the loss of TEMPO was not obvious in the dispersion of either pristine $\mathrm{Au}$ or $\mathrm{Ag}_{2} \mathrm{~S}$ NPs, regardless of the ligand coverage (Supplementary Fig. 7). We, therefore, used ESR to monitor the welding of $\mathrm{Au}$ and $\mathrm{Ag}_{2} \mathrm{~S}$ NPs in situ in dispersion and rule out the possibility of electron beam-accelerated welding of $\mathrm{NP}^{35}$. After different incubation time, an equivalent of the mixture of $\mathrm{Au}$ and $\mathrm{Ag}_{2} \mathrm{~S} \mathrm{NPs}$ was mixed with the same amount of TEMPO and irradiated with visible light. As shown in Fig. 2b, for $\mathrm{Au}$ NPs with low PEG coverage, the loss of TEMPO increased with increasing incubation time, indicating the continuous welding of NPs. The welding proceeded gradually and mostly completed in $\sim 12 \mathrm{~h}$ of incubation, as indicated by a plateau in Fig. 2b. In contrast, no significant change in the loss of TEMPO was observed in the system containing Au NPs with high PEG coverage. This further confirmed that the PEG coverage on NPs plays a crucial role in the occurrence of NP welding.

Taken together, these results suggest an assembly-diffusion mechanism of welding (Fig. 2c). Upon mixing the dispersion of $\mathrm{Au}$ and $\mathrm{Ag}_{2} \mathrm{~S} \mathrm{NPs}$, the weak electrostatic attraction between dissimilar NPs and repulsion between the same types of NPs (i.e., between $\mathrm{Au}$ and $\mathrm{Au}$ NPs or between $\mathrm{Ag}_{2} \mathrm{~S}$ and $\mathrm{Ag}_{2} \mathrm{~S}$ NPs) facilitate the hetero-clustering of NPs. During the incubation, residual citrates weakly bonded on Au NPs gradually desorb to induce conformal contact and atomic diffusion of $\mathrm{Au}$ and $\mathrm{Ag}_{2} \mathrm{~S}$ 

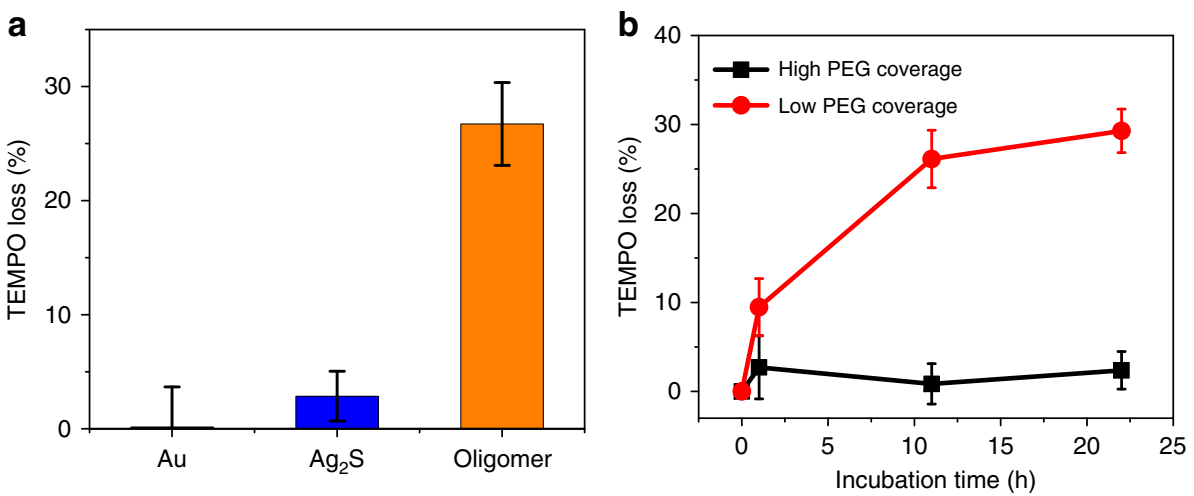

C

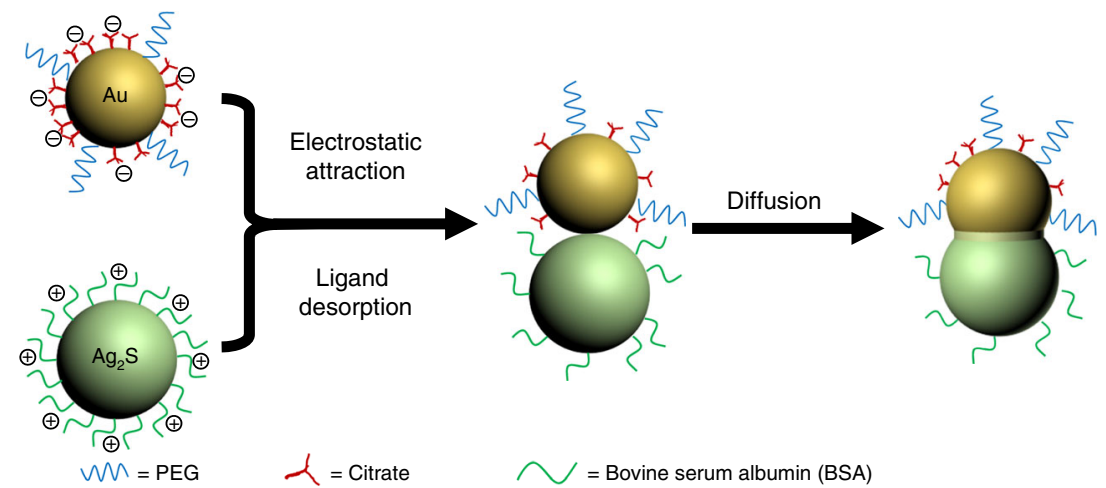

Fig. 2 Mechanism investigation of the welding process using ESR. a A comparison of TEMPO loss in the dispersion of $A u$ NPs, $\mathrm{Ag}_{2} \mathrm{~S} N \mathrm{Ns}_{\mathrm{s}}$ and $\mathrm{Au}-\mathrm{Ag}_{2} \mathrm{~S}$ HNPs, after the irradiation with visible light for $15 \mathrm{~min}$. $\mathbf{b}$ TEMPO loss for incubated $\mathrm{Ag}_{2} \mathrm{~S}$ and $\mathrm{Au} \mathrm{NPs}$ with different polymer coverage as a function of incubation time. Each test is repeated three times. Standard deviations are represented by error bars. c Schematic illustration of the hypothesized welding mechanism

$\mathrm{NPs}^{18}$. The placement of $\mathrm{Au}$ and $\mathrm{Ag}_{2} \mathrm{~S}$ NPs in close proximity initiates atom diffusion between $\mathrm{Au}$ and $\mathrm{Ag}_{2} \mathrm{~S}$ domain and eventually the welding of NPs to produce HNPs ${ }^{35-37}$. The diffusion was supported by the gradual spreading of Au signal from its original spherical shape toward $\mathrm{Ag}_{2} \mathrm{~S}$ domain with gradient signal intensity in energy-dispersive $\mathrm{X}$-ray spectroscopy (EDS) mapping of the $\mathrm{Au}-\mathrm{Ag}_{2} \mathrm{~S} \mathrm{HNP}$ (Supplementary Fig. 8a-d). Unfortunately, the overlap between $\mathrm{Au}$ and $\mathrm{S}$ signal makes it impossible to quantify the distribution of $S$ within the HNPs (Supplementary Fig. 9) ${ }^{38}$. Moreover, X-ray powder diffraction (XRD) results suggested the presence of trace $\mathrm{Ag}_{3} \mathrm{AuS}_{2}$ compounds in the welded HNPs, which further confirms the diffusion of $\mathrm{Au}$ (Supplementary Fig. 10). The corresponding peaks of $\mathrm{Ag}_{3} \mathrm{AuS}_{2}$ were distinctive from those of $\mathrm{Au}$ and $\mathrm{Ag}_{2} \mathrm{~S}$, although they were much weaker than those of $\mathrm{Au}$ and $\mathrm{Ag}_{2} \mathrm{~S}$ due to the very small welding region in individual $\mathrm{Au}-\mathrm{Ag}_{2} \mathrm{~S}$ HNPs.

$\mathrm{Ab}$ initio molecular dynamics simulations were then employed to gain a deeper understanding of the welding mechanism. The two phases were modeled by a slab interface between $(5 \times 3) \mathrm{Ag}_{2} \mathrm{~S}$ $(101)$ and $(8 \times 8) \mathrm{Au}(111)$. Although no obvious welding was observed at reaction temperature $(300 \mathrm{~K})$ probably due to the limited length of simulation time ( $16 \mathrm{ps})$, one can still observe that the $\mathrm{Ag}_{2} \mathrm{~S}$ phase becomes disordered (Fig. 3a, b). Previous studies have suggested that increase of simulation temperature can be used to accelerate the sampling of system with high energy barrier, thus enabling the modeling of slow reactions that are otherwise beyond the time scale of simulation ${ }^{39}$. We, therefore, used a simulation temperature of $800 \mathrm{~K}$ to observe the welding process. High-temperature simulation did confirm the welding phenomenon, with $\mathrm{Au}$ penetrating into the $\mathrm{Ag}_{2} \mathrm{~S}$ matrix, followed by $\mathrm{Ag}$ filling into the empty sites of the Au slab (Fig. 3c). No obvious penetration of S into Au matrix was observed. Moreover, the simulation indicates the diffusion of $\mathrm{Au}$ into $\mathrm{Ag}_{2} \mathrm{~S}$ matrix is easier than Ag into Au matrix (Supplementary Fig. 11), which might be attributed to the more flexible nature of $\mathrm{Ag}_{2} \mathrm{~S}$ layered structure than the close packed fcc Au. Indeed, the barrier for Ag atom running out of $\mathrm{Ag}_{2} \mathrm{~S}$ surface is only $0.80 \mathrm{eV}$, which is much lower than the barrier $(1.45 \mathrm{eV})$ for $\mathrm{Au}$ atom moving out of $\mathrm{Au}$ (111). Combined with the EDS mapping results, we hypothesized that the diffusion of $\mathrm{Au}$ atoms into $\mathrm{Ag}_{2} \mathrm{~S}$ lattice was the primary driving force for the welding process.

The coalescence between $\mathrm{Au}$ and $\mathrm{Ag}_{2} \mathrm{~S}$ may occur via an Ostwald ripening process, which involves the dissolution of $\mathrm{Au}$ $\mathrm{NPs}$ and re-nucleation and growth on the surface of $\mathrm{Ag}_{2} \mathrm{~S} \mathrm{NPs}^{40}$. The ripening process is negligible in our case due to the relative large size of Au NPs (14 nm); however, it could be dominant for $8 \mathrm{~nm} \mathrm{Au}$ NPs which are thermodynamically more unstable (Supplementary Fig. 12). This phenomenon was confirmed by the time-dependent analysis of metal ion concentration by inductively coupled plasma mass spectrometry: the dissolved Au ions significantly increased as a function of time for the $8-\mathrm{nm} \mathrm{NP}$ system, while the concentration of $\mathrm{Au}$ ions in the $14-\mathrm{nm}$ system remained relatively low and constant throughout the welding process (Supplementary Table 2).

Welding of NPs with different shapes and compositions. The welding process was also observed in NPs with distinct shapes and compositions. When shaped NPs were used, the welding process led to the formation of structurally more complex HNPs. For instance, when the dispersion of Au NRs (40 nm in length and $10 \mathrm{~nm}$ in diameter) was mixed with the dispersion of $\mathrm{Ag}_{2} \mathrm{~S}$ NPs, $\mathrm{Ag}_{2} \mathrm{~S}$ NPs preferentially attached to the tips of Au NRs to form matchstick-like HNPs (Fig. 4a, b and Supplementary Fig. 13). We presume that the preferential welding of $\mathrm{Ag}_{2} \mathrm{~S}$ at the ends of $\mathrm{Au}$ NRs is attributed to the deprivation of 


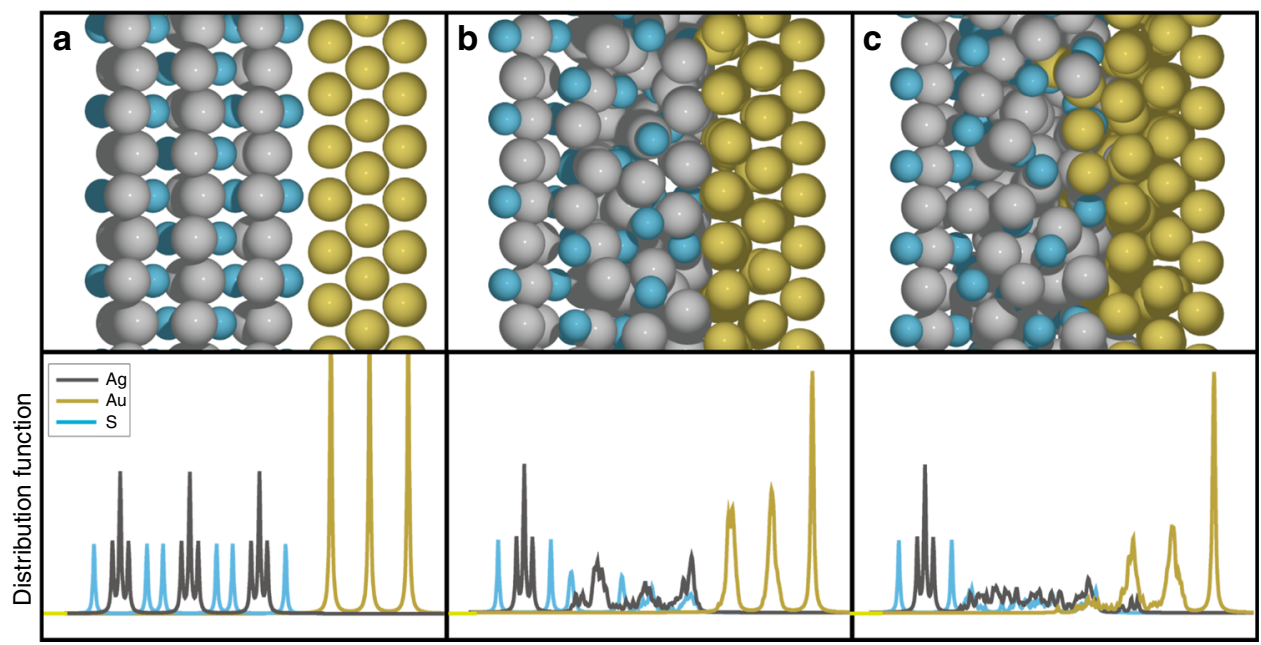

Fig. 3 Molecular dynamics simulation of the welding process. Snapshots (top) and element distribution profiles (bottom) of inter-facial region of the nanostructures a before welding, $\mathbf{b}$ after 16 ps simulation of welding at $300 \mathrm{~K}$, and $\mathbf{c}$ after 16 ps simulation of welding at $800 \mathrm{~K}$. Color: Ag-silver; Au-gold; S-cyan


Fig. 4 Formation of $\mathrm{Au}-\mathrm{Ag}_{2} \mathrm{~S}$ matchstick-like HNPs through welding of $\mathrm{Au}$ NRs and spherical $\mathrm{Ag}_{2} \mathrm{~S} N P s$. a-c TEM images of HNPs obtained by welding Au NRs and $\mathrm{Ag}_{2} \mathrm{~S} N$ Ns at different magnifications. d-f Corresponding FFT pattern of different regions in $\mathbf{c}$ : Au region in red box (d), welding region in blue box $(\mathbf{e})$, and $\mathrm{Ag}_{2} \mathrm{~S}$ region in orange box (f).The red squares, orange circles, and blue triangles in $\mathbf{d}-\mathbf{f}$ highlighted the $\mathrm{FFT}$ patterns of $\mathrm{Au}, \mathrm{Ag}_{2} \mathrm{~S}$, and a new phase, respectively. Scale bars are $100 \mathrm{~nm}$ in $\mathbf{a}, 10 \mathrm{~nm}$ in $\mathbf{b}$, and $5 \mathrm{~nm}$ in $\mathbf{c}$

cetyltrimethylammonium bromide bilayer at the rod ends ${ }^{41}$. This is also supported by the fact that the Au NRs would homogeneously aggregate in an end-to-end manner in the absence of $\mathrm{Ag}_{2} \mathrm{~S}$ NPs (Supplementary Fig. 14). HRTEM image showed lattice fringe of an individual HNP (Fig. 4c): the $0.23 \mathrm{~nm}$ interplanar spacing corresponded to $\mathrm{Au}$ (111) facet and the $0.26 \mathrm{~nm}$ spacing could be assigned to $(-121)$ facet of monoclinic $\mathrm{Ag}_{2} \mathrm{~S}$. In between the $\mathrm{Au}$ and the $\mathrm{Ag}_{2} \mathrm{~S}$ domain, an $\mathrm{Au}$ diffusion region as aforementioned could be clearly observed. The fast Fourier transform (FFT) patterns of this region (Fig. 4e, blue triangles) were distinct from those of $\mathrm{Au}$ (Fig. 4d, red squares) and $\mathrm{Ag}_{2} \mathrm{~S}$ (Fig. 4f, orange circles), indicating the formation a new phase (presumably $\mathrm{Ag}_{3} \mathrm{AuS}_{2}$ ).

When oleylamine $(\mathrm{OAm})$ covered $\mathrm{Au}$ NWs $(8 \mathrm{~nm}$ in diameter $)^{42}$ and spherical $\mathrm{Ag}_{2} \mathrm{~S}$ NPs were used, their welding upon desorption of $\mathrm{OAm}^{19}$ led to the formation of tree burl-like HNPs in which $\mathrm{Ag}_{2} \mathrm{~S}$ NPs selectively attached to the sides of NWs (Fig. 5a, b). Similar to Au NPs and NRs, the diffusion of Au into $\mathrm{Ag}_{2} \mathrm{~S}$ was also observed in HRTEM (Fig. 5c) and EDS mapping (Supplementary Fig. 15). The three-dimensional reconstruction of welded tree burl-like HNPs was obtained by using aberrationcorrected scanning TEM (AC-STEM). TEM images of the 


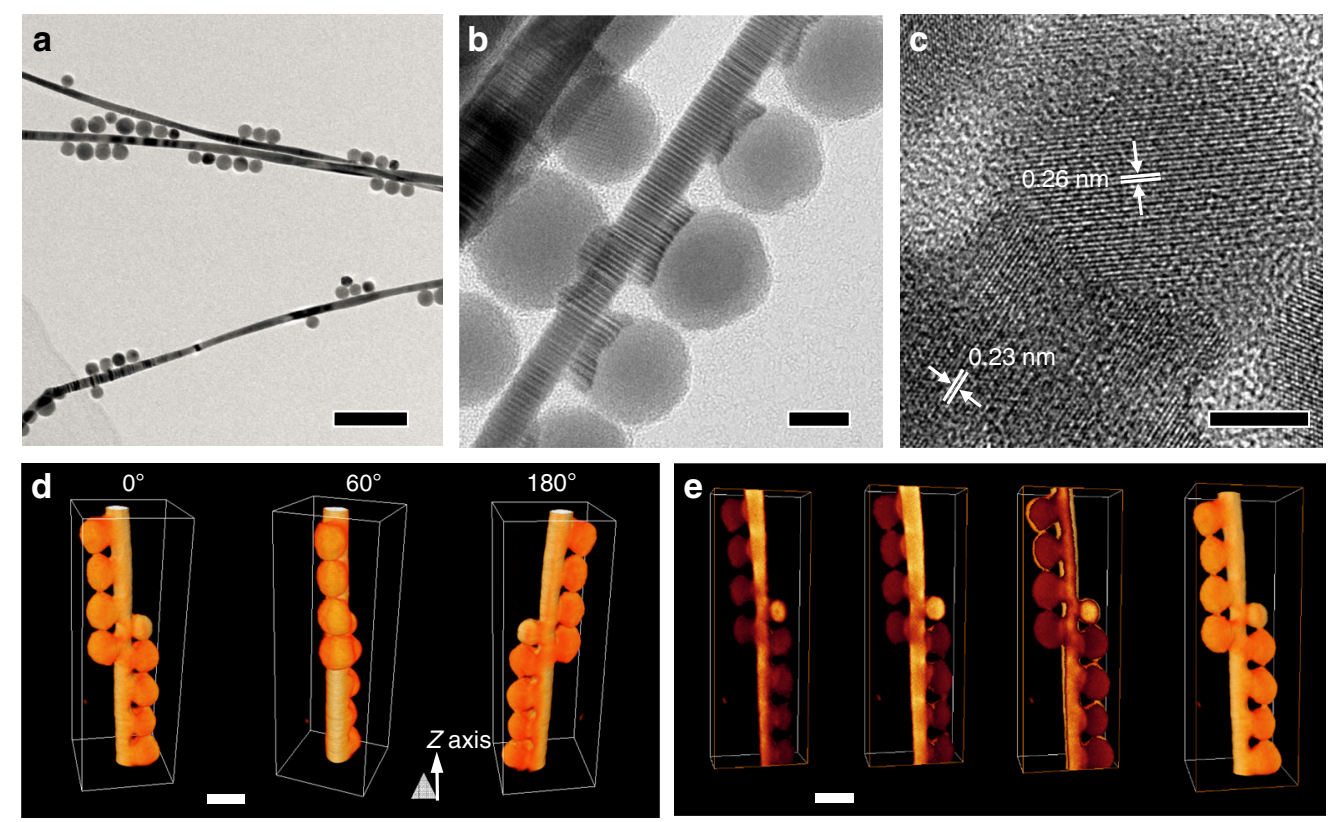

Fig. 5 Formation of $\mathrm{Au}-\mathrm{Ag}_{2} \mathrm{~S}$ tree burl-like HNPs through welding of $\mathrm{Au} N W \mathrm{~N}$ and spherical $\mathrm{Ag}_{2} \mathrm{~S} \mathrm{NPs.} \mathrm{a-c} \mathrm{TEM} \mathrm{images} \mathrm{of} \mathrm{HNPs} \mathrm{obtained} \mathrm{by} \mathrm{welding} \mathrm{Ag} 2 \mathrm{~S}$ NPs and Au NWs at different magnifications. d The volume renderings of the tomographic reconstruction of such HNPs at different rotation angles along $Z$ axis. e Three-dimensional tomographic reconstruction of such $\mathrm{Au}-\mathrm{Ag}_{2} \mathrm{~S}$ HNPs. The progressing cross-sections and the isosurfaces visualize the internal structures of the nanocomposite. Scale bars are $100 \mathrm{~nm}$ in $\mathbf{a}, 10 \mathrm{~nm}$ in $\mathbf{b}, 5 \mathrm{~nm}$ in $\mathbf{c}$, and $20 \mathrm{~nm}$ in $\mathbf{d}$ and $\mathbf{e}$

nanostructure were taken every $2^{\circ}$ when tilting from $-70^{\circ}$ to $+70^{\circ}$ (see Supplementary Movie 1) and used to reconstruct the $3 \mathrm{D}$ model (Fig. 5d). Figure 5e shows the isosurfaces at different cross-sections of the HNPs (see corresponding movie in Supplementary Movie 2), further indicating the welded rather than overlapped structures. Interestingly, the use of ultrathin Au $\mathrm{NWs}$ ( $2 \mathrm{~nm}$ in diameter) led to the simultaneous ripening and welding process (Supplementary Fig. 16).

Selenides such as $\mathrm{Ag}_{2} \mathrm{Se}$ could also weld with $\mathrm{Au}$ NPs in dispersion to form HNPs (Fig. 6). When spherical $\mathrm{Ag}_{2} \mathrm{Se}$ capped with octadecylamine (ODA) was mixed with OAm-capped Au NPs in toluene, similar oligomer-like HNPs could be collected (Fig. 6a). The 0.23 and $0.25 \mathrm{~nm} \mathrm{~d}$-spacing could be assigned to the (111) facet of $\mathrm{Au}$ and the (002) facet of $\mathrm{Ag}_{2} \mathrm{Se}$, respectively (Fig. 6b). We also investigated the welding between spherical $\mathrm{Au}$ NPs and chalcogenides with different shapes. For instance, rodlike $\mathrm{Ag}_{2} \mathrm{~S}$ NPs were prepared use a BSA-directed growth method with modification. Upon mixing with $\mathrm{Au}$ NPs in aqueous dispersion and subsequent incubation, the Au NPs preferentially attached to the sides of $\mathrm{Ag}_{2} \mathrm{~S}$ NRs, forming similar tree burl-like HNPs as in the case of $\mathrm{Au} \mathrm{NW}-\mathrm{Ag}_{2} \mathrm{~S}$ NPs (Fig. 6c). Notably, HRTEM image revealed the diffusion from $\mathrm{Au}$ NPs to $\mathrm{Ag}_{2} \mathrm{~S}$ NRs (Fig. 6d).

\section{Discussion}

In conclusion, we have revealed a unique welding phenomenon between $\mathrm{Au}$ and chalcogenides NPs in dispersion. The surface ligand desorption and Au diffusion are two major criteria that drive the welding process. The welding mechanism was investigated by a combination of tools, including EDS mapping, molecular dynamic simulation, and ESR measurements. We showed that the $\mathrm{Au}$ diffusion-dominated welding process can occur between NPs with distinct shapes or different capping agents or in different solvent media. In current experiments, the welding process produced HNPs with limited control over their nuclearity (i.e., with a defined number of NP precursors fused into a single entity) and the yield of hetero-fused structures (i.e., from distinct NPs) is not sufficiently high. Thus, before the welding process can be utilized for practical synthesis, future efforts should be made on identifying the criteria to improve the product yield and purity of welded HNPs. For instance, better engineering the interactions between NP precursors (e.g., electrostatic attraction between dissimilar NPs and repulsion between same-kind NPs) may lead to high selectivity in the association and fusion of NPs to produce uniform HNPs with controlled morphologies. Nevertheless, the welding process represents an important addition to the existing synthetic approach toward the fabrication of metal-chalcogenide HNPs with well-defined nanostructures, although future efforts are needed to further improve the yield of synthesis. These HNPs may find applications such as solar energy harvesting and conversion, photoluminescence, surface-enhanced Raman scattering, and biological and medical applications.

\section{Methods}

Synthesis of Au NPs. Typically, $10 \mathrm{mg}$ of $\mathrm{HAuCl}_{4}$ was dissolved in $100 \mathrm{ml}$ of $\mathrm{H}_{2} \mathrm{O}$ and heated to boiling under stirring. $3 \mathrm{ml}$ of sodium citrate (1 wt\%) solution was then quickly injected. The reaction mixture was refluxed for $30 \mathrm{~min}$. Au NPs were collected by centrifuging the above solution at $9500 \mathrm{rpm}$ for $15 \mathrm{~min}$ and redispersed in $20 \mathrm{ml}$ of water.

Preparation of PEG-Au NPs. For PEG surface modification of Au NPs with different grafting densities, typically, $5 \mathrm{ml}$ of the abovementioned aqueous dispersion of Au NPs was added in four separate vials, followed by the addition of 10 , 20,40 , and $80 \mu \mathrm{l} \mathrm{of} 1 \mathrm{mg} \mathrm{ml}^{-1}$ fresh prepared PEG-SH aqueous solution, respectively. The dispersions were stirred for $1 \mathrm{~h}$, then incubated at ambient condition for $8 \mathrm{~h}$. The dispersions were then directly used for NP welding. The PEG-Au NPs with different grafting densities were denoted as $\mathrm{Au}-1, \mathrm{Au}-2, \mathrm{Au}-3$, and $\mathrm{Au}-4$, respectively. The original Au NPs were concentrated to the same concentration with $\mathrm{Au}-1$ and denoted as $\mathrm{Au}-0$ for comparison. Detailed synthetic methods for $\mathrm{Au}$ NWs, Au NRs, $\mathrm{Ag}_{2} \mathrm{~S}$ NPs, and $\mathrm{Ag}_{2} \mathrm{Se}$ NPs are shown in Supplementary Methods.

Synthesis of BSA-Ag $\mathbf{2}$ S NPs and NRs. For $\mathrm{Ag}_{2} \mathrm{~S}$ NPs, typically, $25 \mathrm{ml}$ of $10 \mathrm{mM}$ $\mathrm{AgNO}_{3}$ aqueous solution was mixed with $50 \mathrm{ml}$ of $0.2 \mathrm{mg} \mathrm{ml}^{-1}$ BSA aqueous solution in a $250-\mathrm{ml}$ concave flask in an ice bath. The mixture solution was stirred for $30 \mathrm{~min}$ and $25 \mathrm{ml}$ of $10 \mathrm{mM}$ thioacetamide (TAA) aqueous solution was subsequently added. The mixture solution was stirred in an ice bath for another $1 \mathrm{~h}$ 

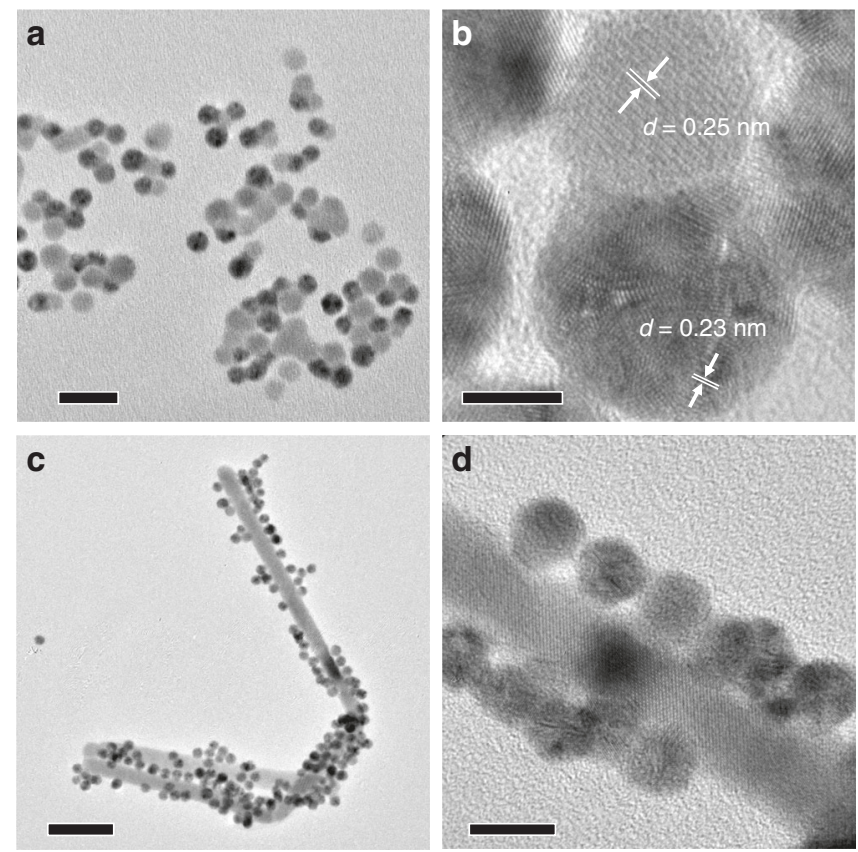

Fig. 6 TEM images showing the welding between Au NPs and different chalcogenide NPs. $\mathbf{a}, \mathbf{c}$ Low and $\mathbf{b}, \mathbf{d}$ high magnification TEM images of $\mathbf{a}, \mathbf{b}$ $\mathrm{Au}-\mathrm{Ag}_{2} \mathrm{Se}$ HNPs welded from spherical $\mathrm{Au}$ and $\mathrm{Ag}_{2} \mathrm{Se} N \mathrm{~N}$ s and $\mathbf{c}$, $\mathbf{d}$ $\mathrm{Au}-\mathrm{Ag}_{2} \mathrm{~S} \mathrm{HNPs}$ welded from spherical $\mathrm{Au}$ and rod-like $\mathrm{Ag}_{2} \mathrm{~S}$ NPs. Scale bars are $20 \mathrm{~nm}$ in $\mathbf{a}, 5 \mathrm{~nm}$ in $\mathbf{b}, 50 \mathrm{~nm}$ in $\mathbf{c}$, and $10 \mathrm{~nm}$ in $\mathbf{d}$

before aging at room temperature for another $24 \mathrm{~h}$. The solution was first centrifuged at $4000 \mathrm{rpm}$ for $20 \mathrm{~min}$, and the solids which were mainly $\mathrm{Ag}_{2} \mathrm{~S}$ NRs were discarded. The supernatant was further centrifuged at $8000 \mathrm{rpm}$ for $20 \mathrm{~min}$, and $\mathrm{Ag}_{2} \mathrm{~S}$ NPs were collected by re-dispersing the solids with $30 \mathrm{ml}$ of water.

For $\mathrm{Ag}_{2} \mathrm{~S} \mathrm{NRs}$, all conditions were same except that the growth solution was aged for 3 days. After that, the $\mathrm{Ag}_{2} \mathrm{~S}$ NRs were collected by centrifuging the solution at $5000 \mathrm{rpm}$ for $15 \mathrm{~min}$.

Detailed synthetic methods for Au NWs, Au NRs, and $\mathrm{Ag}_{2} \mathrm{Se}$ NPs are shown in Supplementary Methods.

Welding of Au and chalcogenide NPs. For the welding of $\mathrm{Au}$ NPs (or NRs) and $\mathrm{Ag}_{2} \mathrm{~S}$ NPs, typically, $0.5 \mathrm{ml}$ of aqueous dispersion of Au NPs (or NRs) was added into a $1.5-\mathrm{ml}$ vial, followed by quick injection of $0.5 \mathrm{ml}$ of aqueous dispersion of $\mathrm{BSA}-\mathrm{Ag}_{2} \mathrm{~S}$ NPs. The mixture dispersion was inversed vigorously and then incubated at room temperature for $24 \mathrm{~h}$. For the welding of $\mathrm{Au} \mathrm{NWs}$ and $\mathrm{Ag}_{2} \mathrm{~S} \mathrm{NPs}$, typically, $0.1 \mathrm{ml}$ of $\mathrm{Au} \mathrm{NWs}$ in toluene was added into $0.8 \mathrm{ml}$ of toluene in a $2-\mathrm{ml}$ vial, followed by quick injection of $0.1 \mathrm{ml}$ of $\mathrm{Ag}_{2} \mathrm{~S}$ NPs in toluene. The mixture dispersion was capped and inversed vigorously, then incubated at room temperature for $48 \mathrm{~h}$. For the welding of OAm-capped $\mathrm{Au} \mathrm{NPs}$ and $\mathrm{Ag}_{2} \mathrm{Se} \mathrm{NPs}$, typically, $0.1 \mathrm{ml}$ of Au NPs in toluene was added into $0.8 \mathrm{ml}$ of toluene in a $2-\mathrm{ml}$ vial, followed by quick injection of $0.1 \mathrm{ml}$ of $\mathrm{Ag}_{2} \mathrm{Se}$ NPs in toluene. The mixture dispersion was capped and inversed vigorously, then incubated at room temperature for $48 \mathrm{~h}$.

Time-dependent ESR test of NP welding. The ESR measurements were carried out using a Bruker EMX ESR spectrometer (Billerica, MA) at ambient temperature. A solar simulator consisting of a $450 \mathrm{~W}$ Xenon lamp filtered to provide simulated sunlight was used in ESR studies. Fifty-microliter aliquots of control or sample dispersions were put into quartz capillary tubes with internal diameters of $0.9 \mathrm{~mm}$ and sealed. The capillary tubes were inserted in the ESR cavity, and the spectra were recorded before and after $15 \mathrm{~min}$ irradiation. All ESR measurements were carried out using the following settings for detection of the spin adducts: $20 \mathrm{~mW}$ microwave power, $100 \mathrm{G}$ scan range, and $1 \mathrm{G}$ field modulation. The sample solution consists of $45 \mu \mathrm{l}$ of NP mixture dispersion and $5 \mu \mathrm{l}$ of $10 \mathrm{uM}$ TEMPO.

Simulations. Ab initio molecular dynamics calculations were done with Vienna Ab-initio Simulation Package (VASP) ${ }^{43,44}$ at generalized-gradient approximation level with exchange-correlation functional in type of $\mathrm{PBE}^{45}$. The interaction between the atomic cores and electrons was described with the projector augmented wave (PAW) method ${ }^{46}$. The valence wave functions were expanded by plane-wave with a cutoff energy of $200 \mathrm{eV}$, within the software suggested ranges. A canonic ensemble was chosen for molecular dynamics with $1.5 \mathrm{fs}$ time step. The $\mathrm{Ag}_{2} \mathrm{~S}$ and $\mathrm{Au}$ interfaces were modeled by $(5 \times 3) \mathrm{Ag}_{2} \mathrm{~S}(101)$ and $(8 \times 8) \mathrm{Au}(111)$ with only $1.2 \%$ unitcell mismatch. Both have a three-layered thickness, with the most outside layer fixed.

Characterizations. UV-Vis extinction measurements were performed using PERKIN LAMBDA $40 \mathrm{UV}-\mathrm{V}$ is system. The structure and morphology of all NPs was analyzed by Hitachi SU-70 Analytical field emission gun SEM (FEG-SEM) operated at $5 \mathrm{kV}$ and JEOL 2100 TEM operated at $200 \mathrm{kV}$ accelerating voltage. HAADF images and EDS mapping were performed on Titan Cubed Themis G2 300 (FEI) using scanning TEM mode under $60 \mathrm{kV}$ accelerating voltage of the electron beam. All the SEM samples were prepared by dispensing a drop of the HNP dispersion on silicon wafers and drying at room temperature. All the TEM samples were prepared by depositing a drop of HNP dispersion onto 300 mesh carboncoated copper grids and allowing solvent evaporation at room temperature.

\section{Data availability}

The data that support the findings of this study are available from the corresponding authors upon reasonable request.

Received: 16 February 2018 Accepted: 5 December 2018 Published online: 15 January 2019

\section{References}

1. Surnev, S., Fortunelli, A. \& Netzer, F. P. Structure-property relationship and chemical aspects of oxide-metal hybrid nanostructures. Chem. Rev. 113, 4314-4372 (2013).

2. Costi, R., Saunders, A. E. \& Banin, U. Colloidal hybrid nanostructures: a new type of functional materials. Angew. Chem. Int. Ed. 49, 4878-4897 (2010).

3. Carbone, L. \& Cozzoli, P. D. Colloidal heterostructured nanocrystals: synthesis and growth mechanisms. Nano Today 5, 449-493 (2010).

4. Cozzoli, P. D., Pellegrino, T. \& Manna, L. Synthesis, properties and perspectives of hybrid nanocrystal structures. Chem. Soc. Rev. 35, 1195-1208 (2006)

5. Huang, Z. et al. Collapsed polymer-directed synthesis of multicomponent coaxial-like nanostructures. Nat. Commun. 7, 12147 (2016).

6. Li, A. et al. Thin heterojunctions and spatially separated cocatalysts to simultaneously reduce bulk and surface recombination in photocatalysts. Angew. Chem. Int. Ed. 55, 13734-13738 (2016).

7. Zhang, Z.-c, Xu, B. \& Wang, X. Engineering nanointerfaces for nanocatalysis Chem. Soc. Rev. 43, 7870-7886 (2014).

8. Xu, C., Wang, B. \& Sun, S. Dumbbell-like $\mathrm{Au}-\mathrm{Fe}_{3} \mathrm{O}_{4}$ nanoparticles for targetspecific platin delivery. J. Am. Chem. Soc. 131, 4216-4217 (2009).

9. Weng, L., Zhang, H., Govorov, A. O. \& Ouyang, M. Hierarchical synthesis of non-centrosymmetric hybrid nanostructures and enabled plasmon-driven photocatalysis. Nat. Commun. 5, 4792 (2014).

10. Jiang, R., Li, B., Fang, C. \& Wang, J. Metal/semiconductor hybrid nanostructures for plasmon-enhanced applications. Adv. Mater. 26, 5274-5309 (2014)

11. Yu, Y., Zhang, Q., Xie, J. \& Lee, J. Y. Engineering the architectural diversity of heterogeneous metallic nanocrystals. Nat. Commun. 4, 1454 (2013).

12. Kwon, S. G. et al. Heterogeneous nucleation and shape transformation of multicomponent metallic nanostructures. Nat. Mater. 14, 215-223 (2015).

13. Xia, Y., Gilroy, K. D., Peng, H.-C. \& Xia, X. Seed-mediated growth of colloidal metal nanocrystals. Angew. Chem. Int. Ed. 56, 60-95 (2017).

14. Zhu, C. et al. Kinetically controlled overgrowth of Ag or Au on Pd nanocrystal seeds: from hybrid dimers to nonconcentric and concentric bimetallic nanocrystals. J. Am. Chem. Soc. 134, 15822-15831 (2012).

15. Feng, Y. et al. An unconventional role of ligand in continuously tuning of metal-metal interfacial strain. J. Am. Chem. Soc. 134, 2004-2007 (2012)

16. DeSantis, C. J., Peverly, A. A., Peters, D. G. \& Skrabalak, S. E. Octopods versus concave nanocrystals: control of morphology by manipulating the kinetics of seeded growth via co-reduction. Nano Lett. 11, 2164-2168 (2011).

17. Tang, Z., Zhang, Z., Wang, Y., Glotzer, S. C. \& Kotov, N. A. Self-assembly of CdTe nanocrystals into free-floating sheets. Science 314 274-278 (2006).

18. Tang, Z., Kotov, N. A. \& Giersig, M. Spontaneous organization of single CdTe nanoparticles into luminescent nanowires. Science 297, 237-240 (2002).

19. Jia, G. et al. Couples of colloidal semiconductor nanorods formed by self limited assembly. Nat. Mater. 13, 301-307 (2014).

20. Boneschanscher, M. P. et al. Long-range orientation and atomic attachment of nanocrystals in 2D honeycomb superlattices. Science 344, 1377-1380 (2014).

21. Schliehe, C. et al. Ultrathin $\mathrm{PbS}$ sheets by two-dimensional oriented attachment. Science 329, 550-553 (2010).

22. Zheng, H. et al. Observation of single colloidal platinum nanocrystal growth trajectories. Science 324, 1309-1312 (2009).

23. Ji, X. et al. Size control of gold nanocrystals in citrate reduction: the third role of citrate. J. Am. Chem. Soc. 129, 13939-13948 (2007). 
24. Grouchko, M. et al. Merging of metal nanoparticles driven by selective wettability of silver nanostructures. Nat. Commun. 5, 2994 (2014).

25. Min Lim, H. et al. Chemical welding of binary nanoparticles: room temperature sintering of CuSe and In2S3 nanoparticles for solution-processed CuInSxSe1-x solar cells. Chem. Commun. 49, 5351-5353 (2013).

26. Mokari, T., Aharoni, A., Popov, I. \& Banin, U. Diffusion of gold into InAs nanocrystals. Angew. Chem. Int. Ed. 45, 8001-8005 (2006).

27. He, J., Liu, Y., Babu, T., Wei, Z. \& Nie, Z. Self-assembly of inorganic nanoparticle vesicles and tubules driven by tethered linear block copolymers. J. Am. Chem. Soc. 134, 11342-11345 (2012).

28. Yang, L. et al. Fabrication of protein-conjugated silver sulfide nanorods in the bovine serum albumin solution. J. Phys. Chem. B 110, 10534-10539 (2006).

29. Rodríguez-Fernández, J., Pastoriza-Santos, I., Pérez-Juste, J., García de Abajo, F. J. \& Liz-Marzán, L. M. The effect of silica coating on the optical response of sub-micrometer gold spheres. J. Phys. Chem. C 111, 13361-13366 (2007).

30. Kalsin, A. M., Kowalczyk, B., Smoukov, S. K., Klajn, R. \& Grzybowski, B. A. Ionic-like behavior of oppositely charged nanoparticles. J. Am. Chem. Soc. 128, 15046-15047 (2006).

31. Grandbois, M., Beyer, M., Rief, M., Clausen-Schaumann, H. \& Gaub, H. E. How strong is a covalent bond? Science 283, 1727-1730 (1999)

32. Pensa, E. et al. The chemistry of the sulfur-gold interface: in search of a unified model. Acc. Chem. Res. 45, 1183-1192 (2012).

33. Schrauben, J. N. et al. Titanium and zinc oxide nanoparticles are protoncoupled electron transfer agents. Science 336, 1298-1301 (2012).

34. He, W. et al. Photogenerated charge carriers and reactive oxygen species in $\mathrm{ZnO} / \mathrm{Au}$ hybrid nanostructures with enhanced photocatalytic and antibacterial activity. J. Am. Chem. Soc. 136, 750-757 (2014).

35. van Huis, M. A. et al. Chemical transformation of Au-tipped CdS nanorods into AuS/Cd core/shell particles by electron beam irradiation. Nano Lett. 11, 4555-4561 (2011).

36. Niu, K.-Y., Liu, M., Persson, K. A., Han, Y. \& Zheng, H. Strain-mediated interfacial dynamics during $\mathrm{Au}-\mathrm{PbS}$ core-shell nanostructure formation. ACS Nano 10, 6235-6240 (2016).

37. Yang, J. \& Ying, J. Y. Diffusion of gold from the inner core to the surface of $\mathrm{Ag}_{2} \mathrm{~S}$ nanocrystals. J. Am. Chem. Soc. 132, 2114-2115 (2010).

38. Lee, S.-U., Hong, J. W., Choi, S.-I. \& Han, S. W. Universal sulfide-assisted synthesis of $\mathrm{M}-\mathrm{Ag}$ heterodimers $(\mathrm{M}=\mathrm{Pd}, \mathrm{Au}, \mathrm{Pt})$ as efficient platforms for fabricating metal-semiconductor heteronanostructures. J. Am. Chem. Soc. 136, 5221-5224 (2014)

39. Liu, J.-C., Wang, Y.-G. \& Li, J. Toward rational design of oxide-supported single-atom catalysts: atomic dispersion of gold on ceria. J. Am. Chem. Soc. 139, 6190-6199 (2017).

40. Qu, J. et al. Coalescence of $\mathrm{Ag}_{2} \mathrm{~S}$ and Au nanocrystals at room temperature. J. Mater. Chem. 21, 11750-11753 (2011).

41. Wang, F., Cheng, S., Bao, Z. \& Wang, J. Anisotropic overgrowth of metal heterostructures induced by a site-selective silica coating. Angew. Chem. Int. Ed. 52, 10344-10348 (2013).

42. Wang, C., Hu, Y., Lieber, C. M. \& Sun, S. Ultrathin Au nanowires and their transport properties. J. Am. Chem. Soc. 130, 8902-8903 (2008).

43. Kresse, G. \& Furthmüller, J. Efficient iterative schemes for ab initio totalenergy calculations using a plane-wave basis set. Phys. Rev. B 54, 11169-11186 (1996).
44. Kresse, G. \& Joubert, D. From ultrasoft pseudopotentials to the projector augmented-wave method. Phys. Rev. B 59, 1758-1775 (1999).

45. Perdew, J. P., Burke, K. \& Ernzerhof, M. Generalized gradient approximation made simple. Phys. Rev. Lett. 77, 3865-3868 (1996).

46. Blöchl, P. E. Projector augmented-wave method. Phys. Rev. B 50, 17953-17979 (1994).

\section{Acknowledgements}

Z.N. gratefully acknowledges the financial support of $3 \mathrm{M}$ Non-tenured Faculty Award. J. G. thanks the National Key R\&D Program of China (2016YFB0600901), and the National Science Foundation of China (21525626, U1463205, and 21761132023). We also acknowledge the support of the Maryland NanoCenter and its AIMLab.

\section{Author contributions}

Z.H., J.G. and Z.N. proposed the concept of this work. Z.H., Q.Z., M.T.P.C. and P.H. conducted the experiments and data analysis. Z.-J.Z. conducted the molecular dynamics simulations. L.H., C.L. and J.L. helped with the EDS mapping and three-dimensional reconstruction. X.J. and J.-J.Y. helped with ESR test. J.G. and Z.N. supervised the research. Z.H., J.G. and Z.N. wrote the manuscript. All authors discussed the results and commented on the manuscript.

\section{Additional information}

Supplementary Information accompanies this paper at https://doi.org/10.1038/s41467018-08206-6.

Competing interests: The authors declare no competing interests.

Reprints and permission information is available online at http://npg.nature.com/ reprintsandpermissions/

Journal peer review information: Nature Communications thanks the anonymous reviewers for their contribution to the peer review of this work.

Publisher's note: Springer Nature remains neutral with regard to jurisdictional claims in published maps and institutional affiliations.

Open Access This article is licensed under a Creative Commons Attribution 4.0 International License, which permits use, sharing, adaptation, distribution and reproduction in any medium or format, as long as you give appropriate credit to the original author(s) and the source, provide a link to the Creative Commons license, and indicate if changes were made. The images or other third party material in this article are included in the article's Creative Commons license, unless indicated otherwise in a credit line to the material. If material is not included in the article's Creative Commons license and your intended use is not permitted by statutory regulation or exceeds the permitted use, you will need to obtain permission directly from the copyright holder. To view a copy of this license, visit http://creativecommons.org/ licenses/by/4.0/.

(C) The Author(s) 2019 